\title{
A Vector Lyapunov Function Characterization of Input-to-State Stability with Application to Robust Global Stabilization of the Chemostat
}

\author{
Iasson Karafyllis $^{1 *}$, Costas Kravaris ${ }^{2 *}$, Lemonia Syrou $^{2}$, and Gerasimos Lyberatos ${ }^{2}$ \\ ${ }^{1}$ Department of Environmental Engineering, Technical University of Crete, 73100 Chania, Greece; ${ }^{2}$ Department of Chemical Engineering, \\ University of Patras, 6500 Patras, Greece
}

This paper develops a novel characterization of uniform input-to-state stability (UISS) in terms of vector Lyapunov functions. The motivation is coming from the problem of robust global stabilization of the chemostat and in particular from anaerobic digestion processes, which are characterized by narrow stability regions and extreme sensitivity to external perturbations, if operated under optimal steady state conditions. The theoretical results enable the derivation of precise robustness margins for a proportional output feedback law that is globally stabilizing the chemostat under nominal conditions. The derivations and results are extended for the case where a feedforward measurement is incorporated in the control law for the chemostat, and it is shown that this leads to improved robustness margins.

Keywords: Lyapunov function methods, robust stability, stabilization

\section{Introduction}

Continuous stirred microbial bioreactors, often called chemostats, cover a wide range of applications; specialised "pure culture" biotechnological processes for the production of specialty chemicals (proteins,

\footnotetext{
*Correspondence to: E-mails: ikarafyl@enveng.tuc.gr;
} kravaris@chemeng.upatras.gr antibiotics etc.) as well as large-scale environmental technology processes of mixed cultures such as wastewater treatment. The dynamics of the chemostat is often adequately represented by a simple dynamic model involving two state variables, the microbial biomass $x$ and the limiting organic substrate $s[2,19]$. For control purposes, two operating variables are usually considered, the dilution rate $D$ which is the manipulated input, and the feed substrate concentration $S_{0}$ which is a load variable. A general model for microbial growth on a limiting substrate in a chemostat is of the form:

$$
\begin{aligned}
& \frac{\mathrm{d} x}{\mathrm{~d} t}=-D x+\mu(s) x \\
& \frac{\mathrm{d} s}{\mathrm{~d} t}=D\left(S_{0}-s\right)-\frac{1}{Y_{x / s}} \mu(s) x
\end{aligned}
$$

where $\mu(s)$ is the specific growth rate, and $Y_{x / s}$ is a biomass yield factor. As will be seen in the next section, one important example is anaerobic digestion, which finds many applications e.g., in wastewater treatment, sludge management, energy from biomass, etc.

In Equation (1), the specific growth rate $\mu(s)$ is given by an empirical correlation, e.g. the Monod equation where $\mu(s)=\mu_{\max } s / K_{S}+s$, or the Haldane equation where $\mu(s)=\mu_{\max } s /\left(K_{S}+s+\left(s^{2} / K_{1}\right)\right)$, or 
the generalized Haldane equation where $\mu(s)=\mu_{\max } s / K_{S}+s\left(1+\left(s / K_{I}\right)^{n}\right), \ldots$ etc., with the parameters $\mu_{\max }, K_{S}, K_{I}, n, \ldots$ all being positive.

In all types of empirical kinetic equations, the function $\mu(s)$ satisfies $\left\{\begin{array}{l}\mu(s)>0 \text { for all } s>0 \\ \mu(0)=0\end{array}\right\}$. However, the specific properties of the function $\mu(s)$ affect the number and stability properties of the chemostat's steady states. For example, in the case of Monod kinetics under constant $D$ and $S_{0}$, there can only be one nontrivial steady state and it is always globally asymptotically stable, but the situation is entirely different in the case of Haldane kinetics. In the next section, in the discussion of the anaerobic digestion process, it will be seen that very meaningful and challenging stabilization problems arise in the case of Haldane kinetics.

The problem of the stabilization of the steady state of the chemostat was considered in [12]. In [6], the problem of the regulation of the chemostat with nonzero mortality rate was considered. In [4], feedback control of the chemostat by manipulating the dilution rate was studied for the promotion of coexistence. Other interesting control studies of the chemostat can be found in [1] and [8]. The stability and robustness of periodic solutions of the chemostat with periodically time-varying dilution rate was studied in [14] in terms of the input-to-state stability (ISS) property. Finally, chemostat models with time delays were considered in $[5,25,26]$.

In this work, we will study the problem of robust global stabilization of a given design steady state $\left(x_{s}^{\mathrm{des}}, s_{s}^{\mathrm{des}}\right)$ of (1), by manipulating the dilution rate $D$, and despite fluctuations in $S_{0}$ and possible errors in the parameters of $\mu(s)$. It will be assumed that the measured output is proportional to the growth rate of the biomass or equivalently the rate of utilization of the substrate:

$$
Q=Y \mu(s) x
$$

where $Y$ is a constant coefficient. It will be seen in the next section that a measurement of the above form is the most meaningful measurement in the anaerobic digestion process.

The feedback stabilization problem for (1) with measured output given by (2), has been studied in [12], where it was shown that the proportional output feedback

$$
D=\frac{1}{Y Y_{x / s}\left(S_{0}-s_{s}^{\mathrm{des}}\right)} Q
$$

is a globally stabilizing feedback. The control law (3) was implemented experimentally in [18] and in [13]. However, the inlet substrate concentration $S_{0}$ was assumed to be a known constant. The robustness property of the closed-loop system (1) with (3) with respect to fluctuations in $S_{0}$ is crucial for the proper operation of the chemostat since fluctuations in $S_{0}$ represent a very common and potentially harmful disturbance.

In this work, precise robustness margins will be derived for the closed-loop system (1) with (3). Subsequently, in order to cover cases where the load variable $S_{0}$ is also measurable, this extra measurement will be incorporated in the control law (3), leading to a feedforward/output feedback controller with improved robustness margin with respect to $S_{0}$. For both types of controllers, robust stability analysis will be in terms of the Uniform ISS (UISS) property and the key mathematical tool will be vector Lyapunov functions.

Vector Lyapunov functions have been proposed recently in [15] for the stability analysis and control of finite-dimensional systems, but have a long history (see the references in [15] and [24]). In this work, we utilize vector Lyapunov functions to develop a novel characterization of the UISS property (see Theorem 3.4 below) that allows the explicit determination of the gain function and can be applied to systems with minimal regularity requirements (see the discussion in Remark 3.5 below). The UISS property was introduced in [21] and had a strong impact in Mathematical Control Theory. The characterization of the UISS property in terms of vector Lyapunov functions that will be developed in the present paper, will allow the proof of UISS property for the closedloop system (1) with (3). In this case, the input is the deviation of the designed value of the feed substrate concentration from its current value $S_{0}$. Because the UISS property holds for the closed-loop system (1) with (3) as long as the deviation in $S_{0}$ is bounded by appropriate constant, the system will keep operating properly despite the deviation and it will be protected from undesirable "shut down". Similar robustness results will be derived under feedforward/feedback control.

Summarizing, the contribution of the present work is:

- a novel characterization of the UISS property in terms of vector Lyapunov functions, which allows the explicit determination of the gain function and can be applied to systems under minimal regularity requirements, 
- the construction of a family of Control Lyapunov Functions for the chemostat that can be used for various control purposes (e.g. sampled-data stabilization of the chemostat, robustness analysis with respect to time-delays),

- a new proof (in terms of control Lyapunov functions) of the fact that (3) is a globally stabilizing feedback for (1),

- the derivation of precise robustness margins with respect to fluctuations of the load variable $S_{0}$ for the closed-loop system (1) with (3),

- the derivation of a feedforward/output feedback controller with improved robustness margin with respect to $S_{0}$

After going over some standard notations, Section 2 will provide some background on anaerobic digestion and it will motivate and explain the control problem for the chemostat in practical terms. Section 3 will develop the theoretical results on a vector Lyapunov function characterization of uniform ISS (UISS). In Section 4, a simple feedback control law will be derived via a Control Lyapunov Function approach, which will guarantee global stability of the chemostat over the entire first quadrant. This feedback control law will be proved to be robust relative to constant and also to time-varying perturbations in the organic load, using the results of Section 3. Finally, Section 5 will develop a feedforward/feedback controller for the chemostat, a robustness analysis will be preformed similarly to Section 4 and it will be seen that incorporation of feedforward action leads to improved robustness margin.

Notations: Throughout this paper we adopt the following notations:

- For a vector $x \in \Re^{n}$ we denote by $|x|$ its usual Euclidean norm and by $x^{\prime}$ its transpose.

- We say that a non-decreasing continuous function $\gamma: \Re^{+} \rightarrow \Re^{+}$is of class $\mathrm{N}$ if $\gamma(0)=0$. We say that an increasing continuous function $\gamma: \Re^{+} \rightarrow \Re^{+}$is of class $K$ if $\gamma(0)=0$. We say that an increasing continuous function $\gamma: \Re^{+} \rightarrow \Re^{+}$is of class $K_{\infty}$ if $\gamma(0)=0$ and $\lim _{s \rightarrow+\infty} \gamma(s)=+\infty$. Clearly, we have $K_{\infty} \subset K \subset \mathrm{N}$. We say that a function $\rho: \Re^{+} \rightarrow \Re^{+}$ is positive definite if $\rho(0)=0$ and $\rho(s)>0$ for all $s>0$. By $K L$ we denote the set of all continuous functions $\sigma=\sigma(s, t): \Re^{+} \times \Re^{+} \rightarrow \Re^{+}$with the properties: (i) for each $t \geq 0$ the mapping $\sigma(\cdot, t)$ is of class $K$; (ii) for each $s \geq 0$, the mapping $\sigma(s, \cdot)$ is non-increasing with $\lim _{t \rightarrow+\infty} \sigma(s, t)=0$.

- Let $D \subseteq \Re^{l}$ be a non-empty set. By $M_{D}$ we denote the class of all Lebesgue measurable and locally bounded mappings $d: \Re^{+} \rightarrow D$.

\section{Practical Motivation: Anaerobic Digestion Processes}

Anaerobic digestion is a complex biochemical process, in which organic compounds are mineralised to biogas (a useful energy product), consisting primarily of methane and carbon dioxide, through a series of reactions mediated by several groups of microorganisms. Under normal (or balanced) operation, the rate of production of the intermediates is matched by their consumption rate; hence there is very little accumulation of these compounds. However, disturbances such as an increase in the concentration of organic compounds in the feed (organic overload), an increase in feed flow rate (hydraulic overload), presence of toxins in the feed, and temperature fluctuations, can cause an imbalance in the process [20], which results in accumulation of volatile organic acids. These acids cause a drop in the $\mathrm{pH}$, inhibiting methanogenesis and the reactor fails. Such a failure has major consequences in the process economics since digester recovery can be a very cumbersome and costly process. For this reason, the development of appropriate control schemes for anaerobic digesters has received significant attention in recent years [16,17].

\subsection{Mathematical Model of Anaerobic Digestion}

For the description of anaerobic digestion, the mathematical model (1) can be used. This system of equations describes methanogenesis, the ultimate step in anaerobic digestion, which is rate limiting and is usually the most sensitive step. In other words, it is assumed that the bioconversion of organics into fatty acids (hydrolysis and acidification) has fast kinetics. Then $x$ and $s$ in (1) represent the methanogen and volatile fatty acid concentrations respectively. The specific growth rate is assumed to follow Haldane kinetics (substrate inhibition) [7]:

$$
\mu(s)=\frac{\mu_{\max } s}{K_{S}+s+\frac{s^{2}}{K_{I}}}
$$

The output of the system is the methane production rate, given by (2), where $Y$ is a yield factor for methane production. The methane production rate $Q$ is easily and reliably measurable on line.

\subsection{Steady States of Anaerobic Digestion}

A first step in the analysis of an anaerobic digester is the calculation of its steady states under a constant dilution rate $D(t)=D_{s}>0$. Apart from the trivial 
steady state $\left(x_{s}=0, s_{s}=S_{0}\right)$ that corresponds to washout of the biomass, the steady states of an anaerobic digester are calculated from the set of equations:

$$
\left\{\begin{array}{l}
\mu\left(s_{s}\right)=D_{s} \\
x_{s}=Y_{x / s}\left(S_{0}-s_{s}\right)
\end{array}\right\}
$$

with only positive solutions $\left(x_{s}>0\right.$ and $\left.s_{s}>0\right)$ being acceptable on physical grounds.

When the specific growth rate follows equation (4) and the dilution rate $0<D_{s} \leq D_{\max }$, where

$$
D_{\max }=\left\{\begin{array}{l}
\frac{\mu_{\max } \cdot S_{0}}{K_{s}+S_{0}+\frac{S_{0}^{2}}{K_{I}}}, \text { if } S_{0} \leq \sqrt{K_{s} \cdot K_{I}} \\
\frac{\mu_{\max }}{1+2 \cdot \sqrt{\frac{K_{s}}{K_{I}}}}, \text { if } S_{0} \geq \sqrt{K_{s} \cdot K_{I}}
\end{array}\right.
$$

the set of equations (5) admits one or two positive solutions; for dilution rates higher than $D_{\max }$, the set of equations (5) does not admit any solution at all. Two positive steady states occur in the case $S_{0}>\sqrt{K_{S} \cdot K_{I}}$ (organic feed in excess) and for dilution rate $\frac{\mu_{\max } \cdot S_{0}}{K_{s}+S_{0}+\frac{S_{0}^{2}}{K_{I}}}<D_{s}<\frac{\mu_{\max }}{1+2 \cdot \sqrt{\frac{K_{s}}{K_{I}}}}=D_{\max }$

In practice, anaerobic digesters operate under excess of organic feed and under not too low dilution rate, therefore the presence of two steady states is the most common situation.

\subsection{Local Asymptotic Stability}

The eigenvalues of the linearization of (1) around a nontrivial steady state are given by:

$$
\begin{aligned}
& \lambda_{1}=-\mu\left(s_{s}\right)=-D_{s} \\
& \lambda_{2}=-\left[\frac{d \mu}{d s}\left(s_{s}\right)\right]\left(S_{0}-s_{s}\right)
\end{aligned}
$$

For positive dilution rate $\left(D_{s}>0\right)$ and for a positive steady state $\left(x_{s}>0 \Rightarrow s_{s}<S_{0}\right)$, local asymptotic stability is guaranteed as long as $\frac{d \mu}{d s}\left(s_{s}\right)>0$.

When the specific growth rate follows equation (4), the stability condition translates to $s_{s}<\sqrt{K_{s} \cdot K_{I}}$. In case there are two positive steady states, it can be shown that one of them satisfies $s_{S}<\sqrt{K_{S} \cdot K_{I}}$, hence it is stable, whereas the other has $s_{s}>\sqrt{K_{s} \cdot K_{I}}$, hence it is unstable. Comparing the two steady states corresponding to the same value of the dilution rate, one can see that the methane production rate is higher at the stable steady state, and this makes the stable steady state more desirable from a performance point of view.

\subsection{Optimal Steady State for Maximal Methane Production}

For the purpose of optimal design of the operating steady state conditions of an anaerobic digester, a meaningful criterion is the maximization of the methane production rate. At steady state, the methane production rate is calculated from:

$$
\begin{aligned}
Q_{s} & =Y \mu\left(s_{s}\right) x_{s} \\
& =Y Y_{x / s} \mu\left(s_{s}\right)\left(S_{0}-s_{s}\right)
\end{aligned}
$$

and the maximum is calculated from:

$$
\frac{d Q_{s}}{d s_{s}}=0 \Leftrightarrow\left[\frac{d \mu}{d s}\left(s_{s}\right)\right]\left(S_{0}-s_{s}\right)-\mu\left(s_{s}\right)=0
$$

Substituting the expression for $\mu(s)$ given by (4), the optimal steady state is found to be:

$$
s_{s}^{\mathrm{opt}}=\frac{S_{0}}{1+\sqrt{1+\frac{S_{0}}{K_{S}}\left(1+\frac{S_{0}}{K_{I}}\right)}}
$$

and this corresponds to biomass

$$
x_{s}^{\mathrm{opt}}=Y_{x / s}\left(S_{0}-s_{s}^{\mathrm{opt}}\right)=\frac{Y_{x / s} S_{0}}{1+\frac{1}{\sqrt{1+\frac{S_{0}}{K_{S}}\left(1+\frac{S_{0}}{K_{I}}\right)}}}
$$

and dilution rate

$$
D_{s}^{\mathrm{opt}}=\frac{\mu_{\mathrm{max}} \cdot s_{s}^{\mathrm{opt}}}{K_{s}+s_{s}^{\mathrm{opt}}+\frac{\left(s_{s}^{\mathrm{opt}}\right)^{2}}{K_{I}}}
$$

It can be easily shown that the optimal steady state is locally asymptotically stable.

For the following values of the parameters:

$$
\begin{aligned}
S_{0} & =10,000 \mathrm{mg} / 1, K_{S}=100 \mathrm{mg} / 1, \mu_{\max }=0.5 d^{-1}, \\
K_{I} & =4,000 \mathrm{mg} / 1, Y_{x / s}=0.05 \mathrm{mg} / \mathrm{mg}, \\
Y & =0.00746 l_{\text {reactor }}^{-1} d^{-1}
\end{aligned}
$$

which are representative of a typical anaerobic digestion process, the optimal steady state from equation (6) is $s_{s}=506.714 \mathrm{mg} / l$. This corresponds to $x_{S}^{\mathrm{opt}}=474.664 \mathrm{mg} / l, \quad D_{S}^{\mathrm{opt}}=0.377633 d^{-1} \quad$ and $Q_{S}=1.337205 l_{\text {reactor }}^{-1} d^{-1}$.

The above numerical values of the parameters and the resulting optimal steady state conditions will be used in the numerical calculations throughout this paper. 


\subsection{The Need for Control}

Figure 1 depicts the phase portrait of the system dynamics under constant dilution rate $D$, in particular for the optimal value $D=D_{S}^{\mathrm{opt}}=0.377633 d^{-1}$, which corresponds to maximal methane production rate. For this value of the dilution rate, there are three steady states for the system:

1. The trivial steady state point $\left(x_{s}=0, s_{s}=S_{0}\right)$ that corresponds to washout: it is a locally asymptotically stable equilibrium point.

2. An unstable steady state point in the interior of the first quadrant: it is a saddle point with a stable manifold.

3. A stable steady state point in the interior of the first quadrant: it is a locally asymptotically stable equilibrium point and it is the operating steady state point that maximizes the methane production rate.

In the diagram, the points $\mathrm{S}$ and $\mathrm{U}$ represent the stable and unstable steady states, respectively in the interior of the first quadrant. The stability regions of the washout steady state and the steady state $\mathrm{S}$ are separated by a curve (separatrix), which is the graph of the stable manifold for the unstable steady state $\mathrm{U}$. Notice that the optimal steady state $\mathrm{S}$ is locally stable but the stability region is very small (in fact the stability region of the washout steady state is "larger" than the stability region of the desired steady state). This makes the optimal operation of the biochemical reactor very sensitive to disturbances.

The goal of control is the stabilization of the system in the sense of enlargement of the stability region.

\section{Vector Lyapunov Function Characterization of Uniform ISS}

The notion of UISS was proposed in [21]. Consider the system:

$$
\begin{aligned}
& \dot{x}=f(d, x, u) \\
& x \in \Re^{n}, d \in \Omega, u \in U \subseteq \Re^{m}
\end{aligned}
$$

where $\Omega \subseteq \Re^{l}$ is compact, $U \subseteq \Re^{m}$ is a non-empty set with $0 \in U$ and $f$ is a continuous vector field with $f(d, 0,0)=0$ for all $d \in \Omega$. Furthermore, there exists a symmetric positive definite matrix $P \in \Re^{n \times n}$ such that for every bounded $S \subset \Re^{n} \times U$, there exists a constant $L \geq 0$ satisfying the following inequality:

$$
\begin{aligned}
& (x-y)^{\prime} P(f(d, x, u)-f(d, y, u)) \leq L|x-y|^{2} \\
& \forall(x, u, y, u) \in S \times S, \forall d \in \Omega
\end{aligned}
$$

It follows that for every $\left(x_{0}, u, d\right) \in \Re^{n} \times M_{U} \times M_{D}$ there exists a unique solution to the initial value problem (7) with $x(0)=x_{0} \in \Re^{n}$ corresponding to input $(u, d) \in M_{U} \times M_{\Omega}$.

Definition 3.1: Consider the control system (7) and suppose that there exist functions $\sigma \in K L, \gamma \in \mathrm{N}$ such that the following estimate holds for all $\left(x_{0}, u, d\right) \in$ $\Re^{n} \times M_{U} \times M_{\Omega}$ and $t \geq 0$ :

$$
|x(t)| \leq \sigma\left(\left|x_{0}\right|, t\right)+\sup _{0 \leq \tau \leq t} \gamma(|u(\tau)|)
$$

where $x(t)$ denotes the solution of (7) with initial condition $x(0)=x_{0} \quad$ corresponding to input $(u, d) \in$ $M_{U} \times M_{\Omega}$. Then we say that (7) satisfies the UISS property with gain $\gamma \in N$ from the input $u \in M_{U}$.

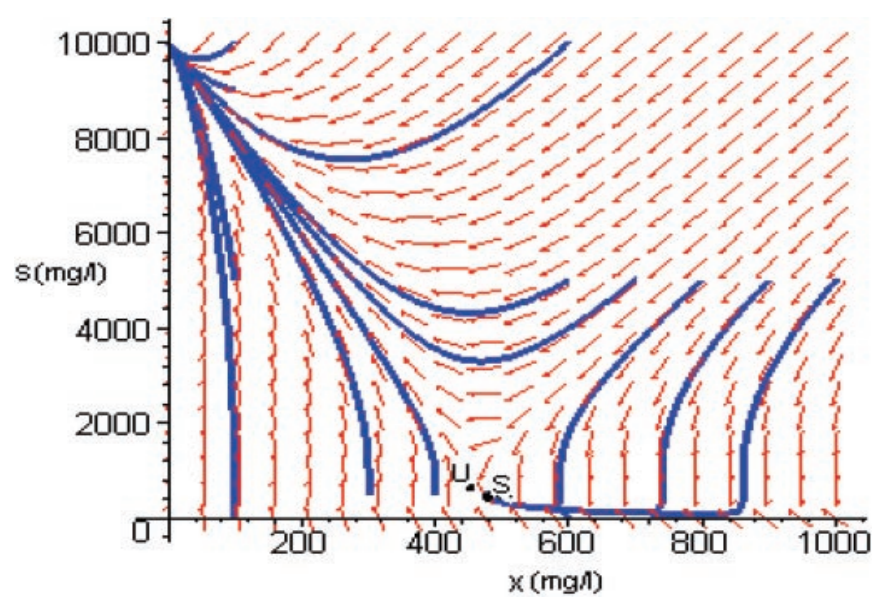

Fig. 1. Phase portrait of the open-loop dynamics. 
Although the above property was introduced in [21] under the name ISS, we prefer the name UISS because various extensions of this notions have been proposed in the literature (non-UISS, weighted ISS, etc.-see [11] and references therein).

For the proof of the vector Lyapunov function characterization of the UISS property we will need the following two technical lemmas. The following lemma is a direct corollary of Theorem 1 in [23].

Lemma 3.2: For every $\sigma \in K L$ and $a \in N$ with $a(s)<s$ for all $s>0$, there exists $\tilde{\sigma} \in K L$ with the following property: if $y:\left[t_{0}, t_{1}\right) \rightarrow \Re^{+}, u: \Re^{+} \rightarrow \Re^{+}$are locally bounded functions and $M \geq 0$ a constant such that the following inequality holds for all $\xi \in\left[t_{0}, t_{1}\right)$ :

$$
\begin{gathered}
y(t) \leq \max \left\{\sigma(M, t-\xi) ; a\left(\sup _{\xi \leq \tau \leq t} y(\tau)\right) ; u(t)\right\}, \\
\forall t \in\left[\xi, t_{1}\right)
\end{gathered}
$$

then the following estimate holds for all $t \in\left[t_{0}, t_{1}\right)$ :

$$
y(t) \leq \max \left\{\tilde{\sigma}\left(M, t-t_{0}\right) ; \sup _{t_{0} \leq \tau \leq t} u(\tau)\right\}, \forall t \in\left[t_{0}, t_{1}\right) .
$$

The second technical lemma is a comparison lemma, which provides a sharp estimate of the evolution of Lyapunov functions (compare the obtained estimate with Theorem 5.2, page 218 in [9]). Its proof can be found in [10].

Lemma 3.3: For each positive definite continuous function $\rho: \Re^{+} \rightarrow \Re^{+}$there exists a function $\sigma$ of class $K L$, with $\sigma(s, 0)=s$ for all $s \geq 0$ with the following property: if $y:\left[t_{0}, t_{1}\right] \rightarrow \Re^{+}$is an absolutely continuous function, $u: \Re^{+} \rightarrow \Re^{+}$is a locally bounded mapping and $I \subset\left[t_{0}, t_{1}\right]$ a set of Lebesgue measure zero such that $\dot{y}(t)$ is defined on $\left[t_{0}, t_{1}\right] \backslash I$ and such that the following implication holds for all $t \in\left[t_{0}, t_{1}\right] \backslash I$ :

$$
y(t) \geq u(t) \Rightarrow \dot{y}(t) \leq-\rho(y(t))
$$

then the following estimate holds for all $t \in\left[t_{0}, t_{1}\right]$ :

$$
y(t) \leq \max \left\{\sigma\left(y\left(t_{0}\right), t-t_{0}\right), \sup _{t_{0} \leq s \leq t} \sigma(u(s), t-s)\right\}
$$

We are now in a position to state and prove our main result concerning the vector Lyapunov function characterization of the UISS property.
Theorem 3.4. (Vector Lyapunov Function Characterization of the UISS Property): Consider system (7) and suppose that there exists a family of functions $V_{i} \in C^{1}\left(\Re^{n} ; \Re^{+}\right) \quad(i=1, \ldots, k), \quad$ functions $a_{1}, a_{2} \in$ $K_{\infty} a, \zeta \in N$ with $a(s)<s$ for all $s>0$ and a family of positive definite functions $\rho_{i} \in C^{0}\left(\Re^{+} ; \Re^{+}\right)$ $(i=1, \ldots, k)$, such that:

$$
a_{1}(|x|) \leq \max _{i=1, \ldots, k} V_{i}(x) \leq a_{2}(|x|), \forall x \in \Re^{n}
$$

and for every $i=1, \ldots, k$ and $(x, u) \in \Re^{n} \times U$ the following implication holds:

$$
\begin{aligned}
& " \text { If } \max \left\{\zeta(|u|), \max _{j=1, \ldots, k} a\left(V_{j}(x)\right)\right\} \leq V_{i}(x) \\
& \text { then } \sup \left\{\nabla V_{i}(x) f(d, x, u): d \in D\right\} \leq-\rho_{i}\left(V_{i}(x)\right) "
\end{aligned}
$$

Then system (7) satisfies the UISS property with gain $\gamma=a_{1}^{-1} \circ \zeta \in N$ from the input $u \in M_{u}$.

Proof: Consider a solution $x(t)$ of (7) corresponding to arbitrary $(u, d) \in M_{U} \times M_{\Omega}$ with initial condition $x(0)=x_{0} \in \Re^{n}$. Clearly, there exists a maximal existence time for the solution denoted by $t_{\max } \leq+\infty$. Let $V_{i}(t)=V_{i}(x(t)), \quad i=1, \ldots, k$, absolutely continuous functions on $\left[0, t_{\max }\right)$. Moreover, let $V(t):=\max _{i=1, \ldots, k} V_{i}(t)$ and $I \subset\left[0, t_{\max }\right)$ be the zero Lebesgue measure set where $x(t)$ is not differentiable or $\dot{x}(t) \neq f(d(t), x(t), u(t))$. By virtue of (14), it follows that the following implication holds for $t \in\left[0, t_{\max }\right) \backslash I$ and $i=1, \ldots, k$ :

$$
\begin{aligned}
V_{i}(t) & \geq \max \{\zeta(|u(t)|) ; a(V(t))\} \\
\Rightarrow \dot{V}_{i}(t) & \leq-\rho_{i}\left(V_{i}(t)\right)
\end{aligned}
$$

Lemma 3.3 implies that there exists a family of continuous functions $\sigma_{i}$ of class $K L(i=1, \ldots, k)$, with $\sigma_{i}(s, 0)=s$ for all $s \geq 0$ such that for all $\xi \in\left[0, t_{\max }\right)$, $t \in\left[\xi, t_{\max }\right)$ and $i=1, \ldots, k$ we have:

$$
\begin{aligned}
& V_{i}(t) \leq \max \\
& \left\{\begin{array}{l}
\sigma_{i}\left(V_{i}(\xi), t-\xi\right) ; \sup _{\xi \leq \tau \leq t} \sigma_{i}(a(V(\tau)), t-\tau) ; \\
\sup _{\xi \leq \tau \leq t} \sigma_{i}(\zeta(|u(\tau)|), t-\tau)
\end{array}\right\}
\end{aligned}
$$

Let $\sigma(s, t):=\max _{i=1, \ldots, k} \sigma_{i}(s, t)$, which is a function of class $K L$ that satisfies $\sigma(s, 0)=s$ for all $s \geq 0$. An 
immediate consequence of definition $V(t):=$ $\max _{i=1, \ldots, k} V_{i}(t)$, estimate (16) with $\xi=0$ and the fact that $i=1, \ldots, k$
$\sigma_{i}(s, 0)=s$ for all $s \geq 0(i=1, \ldots, k)$ is the following estimate, which holds for all $t \in\left[0, t_{\max }\right)$ :

$$
\begin{aligned}
V(t) \leq & \max \left\{\sigma(V(0), t) ; a\left(\sup _{0 \leq s \leq t} V(s)\right) ;\right. \\
& \left.\sup _{0 \leq s \leq t} \zeta(|u(s)|)\right\}
\end{aligned}
$$

Inequality (17) and the fact that $\sigma(s, 0)=s$ for all $s \geq 0$, imply the following inequality:

$$
\begin{aligned}
\sup _{0 \leq s \leq t} V(s) \leq & \max \left\{V(0) ; a\left(\sup _{0 \leq s \leq t} V(s)\right)\right. \\
& \left.\sup _{0 \leq s \leq t} \zeta(|u(s)|)\right\} \text {, for all } t \in\left[0, t_{\max }\right)
\end{aligned}
$$

Making use of the fact that $a(s)<s$ for all $s>0$, we obtain from (18):

$$
\begin{gathered}
V(t) \leq \max \left\{V(0) ; \sup _{0 \leq s \leq t} \zeta(|u(s)|)\right\}, \\
\text { for all } t \in\left[0, t_{\max }\right)
\end{gathered}
$$

Clearly, inequality (19) implies that as long as the solution of (7) exists, $V(t)$ is bounded. A standard contradiction argument in conjunction with inequality (13) shows that necessarily we must have $t_{\max }=+\infty$. We conclude that estimate (16) holds for all $\xi \geq 0$, $t \geq \xi$ and $i=1, \ldots, k$. Similarly, estimate (19) holds for all $t \geq 0$.

An immediate consequence of estimate (16), definitions $V(t):=\max _{i=1, \ldots, k} V_{i}(t), \quad \sigma(s, t):=\max _{i=1, \ldots, k} \sigma_{i}(s, t)$ and the fact that $\sigma_{i}(s, 0)=s$ for all $s \geq 0(i=1, \ldots, k)$ is the following estimate, which holds for all $t \geq \xi \geq 0$ :

$$
\begin{gathered}
V(t) \leq \max \left\{\sigma(V(0), t-\xi) ; a\left(\sup _{\xi \leq s \leq t} V(s)\right) ;\right. \\
\left.\sup _{0 \leq s \leq t} \zeta(|u(s)|)\right\}
\end{gathered}
$$

Lemma 3.2 in conjunction with inequality (20) implies the existence of $\bar{\sigma} \in K L$ such that:

$$
V(t) \leq \max \left\{\bar{\sigma}(V(0), t) ; \sup _{0 \leq s \leq t} \zeta(|u(s)|)\right\}
$$

Estimate (21) in conjunction with inequality (13) shows that (7) satisfies the UISS property with gain $\gamma=a_{1}^{-1} \circ \zeta \in N$ from the input $u \in M_{U}$. The proof is complete.
Remark 3.5: Theorem 3.4 allows the explicit determination of the gain function $\left(\gamma=a_{1}^{-1} \circ \zeta \in N\right)$ for the ISS property. However, there are other results in the literature that utilize vector Lyapunov functions and guarantee the UISS property under weaker conditions, like in [3]. A slight generalization of the analysis in [3] would lead to the following result, although it is not explicitly stated in their paper.

Theorem 3.6 (Alternative Vector Lyapunov Function Characterization of the UISS property): Consider system $\dot{x}=f(x, u),(x, u) \in \Re^{n} \times \Re^{m}$ where $f: \Re^{n} \times$ $\Re^{m} \rightarrow \Re^{n}$ is a locally Lipschitz vector field with $f(0,0)=0$ and suppose that there exists a family of functions $V_{i} \in C^{1}\left(\Re^{n} ; \Re^{+}\right) \quad(i=1, \ldots, k)$, functions $a_{1}, a_{2}, \varphi \in K_{\infty} \quad \zeta \in N, \quad \gamma_{i, j} \in N \quad(i, j=1, \ldots, k)$ with $\max _{i=1, \ldots, k}\left(s_{i}-\sum_{j \neq i} \gamma_{i, j}\left(s_{j}+\varphi\left(s_{j}\right)\right)\right)>0$ for all $s_{1}, \ldots, s_{k} \geq 0$ with $\max _{i=1, \ldots, k}\left(s_{i}\right)>0$ and a family of positive definite functions $\rho_{i} \in C^{0}\left(\Re^{+} ; \Re^{+}\right)(i=1, \ldots, k)$, such that:

$$
a_{1}(|x|) \leq \max _{i=1, \ldots, k} V_{i}(x) \leq a_{2}(|x|), \forall x \in \Re^{n}
$$

and for every $i=1, \ldots, k$ and $(x, u) \in \Re^{n} \times U$ the following implication holds:

$$
\begin{gathered}
\text { "If } \zeta(|u|)+\sum_{j \neq i} \gamma_{i, j}\left(V_{j}(x)\right) \leq V_{i}(x) \\
\text { then } \nabla V_{i}(x) f(x, u) \leq-\rho_{i}\left(V_{i}(x)\right) "
\end{gathered}
$$

Then system $\dot{x}=f(x, u),(x, u) \in \Re^{n} \times \Re^{m}$ satisfies the UISS property from the input $u \in M_{U}$.

A comparison between Theorems 3.4 and 3.6 is useful. Although condition (14) is much more demanding than (23), the result of Theorem 3.6 does not provide an explicit determination of the gain function. Furthermore, it is restricted to systems without disturbances and demands Lipschitz continuity in both the input and the state.

\section{Robust Feedback Stabilization of the Chemostat}

Consider the dynamic system (1) with $D \in$ $[0,+\infty), x \in(0,+\infty), s \in(0,+\infty)$ where $\mu: \Re^{+} \rightarrow \Re^{+}$ is a smooth function with $\mu(0)=0, \mu(s)>0$ for all $s>0$. The reader should notice that the closed first quadrant in $\Re^{2}$, i.e., the set $[0,+\infty) \times[0,+\infty)$, is positively invariant for system (1) for any measurable and locally essentially bounded control $D: \Re^{+} \rightarrow \Re^{+}$. This can be verified by the inequalities $\dot{s}>0$ when $s=0$ and $\dot{x} \geq 0$, when $x=0$ (i.e., for every measurable and locally essentially bounded control $D: \Re^{+} \rightarrow \Re^{+}$, 
the closed first quadrant in $\Re^{2}$ is a viability domain for system (1)). Consequently, for every measurable and locally essentially bounded control $D: \Re^{+} \rightarrow \Re^{+}$and for every $(x(0), s(0)) \in[0,+\infty) \times[0,+\infty)$, the solution of (1) with initial condition $(x(0), s(0)) \in[0,+\infty)$ $\times[0,+\infty)$ corresponding to the control input $D: \Re^{+} \rightarrow \Re^{+}$satisfies $(x(t), s(t)) \in[0,+\infty) \times[0,+\infty)$ as long as the solution exists. Notice that the closed first quadrant in $\Re^{2}$ is the set where the state variables of (1) are physically meaningful. However, the boundary of the closed first quadrant in $\Re^{2}$, i.e., the semi-axes $x=0$ and $s=0$, contain operating points of the chemostat that must be avoided (since they correspond to zero growth rate and, in particular, in the case of anaerobic digestion, to zero methane production rate). Therefore, we are interested only in solutions of (1) that lie in the open first quadrant in $\Re^{2}$.

Also, consider the coordinate transformation:

$$
x_{1}=\ln \left(\frac{x}{x_{s}^{\mathrm{des}}}\right) ; \quad x_{2}=\ln \left(\frac{S}{s_{s}^{\mathrm{des}}}\right)
$$

where $x_{s}^{\text {des }}, s_{s}^{\text {des }}$ are the design steady state values for $x$, $s$, respectively (e.g., in anaerobic digestion, the optimal steady state of Section 2.4 given by equation (6), or any other design steady state arising from other criteria). Transformation (24) maps the open first quadrant onto $\Re^{2}$. In particular, it maps the design steady state $\left\{\begin{array}{l}x_{s}=Y_{x / s}\left(S_{0}-s_{s}^{\text {des }}\right) \\ s_{s}=s_{s}^{\text {des }}\end{array}\right\}$ to the origin.

Under the transformation (24), the system (1) becomes:

$$
\begin{aligned}
& \dot{x}_{1}=\mu\left(s_{s}^{\mathrm{des}} \mathrm{e}^{x_{2}}\right)-D \\
& \dot{x}_{2}=D\left(\frac{S_{0}}{s_{s}^{\mathrm{des}}} \mathrm{e}^{-x_{2}}-1\right)-\mu\left(s_{s}^{\mathrm{des}} \mathrm{e}^{x_{2}}\right)\left(\frac{S_{0}}{s_{s}^{\mathrm{des}}}-1\right) \mathrm{e}^{x_{1}-x_{2}}
\end{aligned}
$$

The above state-space description is more convenient for analysis purposes since the state space is now the entire $\Re^{2}$, the objective being to stabilize the system at the origin.

\subsection{Controllability Analysis}

A straightforward calculation can show that the linearization of system (25) at the desired equilibrium point $\left(x_{1}, x_{2}\right)=(0,0)$ is not controllable. It will now be shown that system (25) is not a controllable system, even in a nonlinear sense (see [22]). Particularly, we have:

Proposition 4.1: There is no $T>0$ such that system (25) is controllable in time $T$.
Proof: Notice that controllability in time $T$ requires that for every vectors $\xi, \zeta \in \Re^{2}$, there is a measurable and locally essentially bounded control $D: \Re^{+} \rightarrow$ $[0,+\infty)$, such that the solution of (25) with initial condition $x(0)=\xi$ corresponding to the control $D: \Re^{+} \rightarrow[0,+\infty)$ satisfies $x(T)=\zeta$. Indeed, let $\xi=\left(\begin{array}{l}\xi_{1} \\ \xi_{2}\end{array}\right) \neq 0 \in \Re^{2}$ with $e^{\xi_{2}}+\left(\frac{S_{0}}{S_{S} d e s}-1\right) e^{\xi_{1}}-\frac{S_{0}}{s_{S}} \neq 0$ and $\zeta=0 \in \Re^{2}$. Suppose that there exists a control $D: \Re^{+} \rightarrow[0,+\infty)$ such that the solution of (25) with initial condition $x(0)=\xi$ corresponding to the control $D:[0, T] \rightarrow[0,+\infty)$ satisfies $x(T)=0$. Consider the function $W(t)=\mathrm{e}^{x_{2}(t)}+\left(\frac{S_{0}}{s_{S}^{d e s}}-1\right) \mathrm{e}^{x_{1}(t)}-\frac{S_{0}}{s_{S}^{d e s}}$. The derivative of $W(t)$ exists almost everywhere on $[0, T]$ and satisfies $\dot{W}(t)=-D(t) W(t)$ almost everywhere on $[0, T]$. It follows that $W(t)=\mathrm{e}^{-\int_{0}^{t} D(\tau) \mathrm{d} \tau} W(0)$ for all times in $[0, T]$. However this implies $0=W(T)=\mathrm{e}^{-\int_{0}^{T} D(t) \mathrm{d} t} W(0) \neq 0$, a contradiction. The proof is complete.

\subsection{Synthesis of a Globally Stabilizing Control Law}

Although there is no control that can stir system (25) to the desired equilibrium in finite time (in general), it is possible that we can find controls that stir system (25) to the desired equilibrium asymptotically. Particularly, the controls can be given in feedback form, which guarantees robustness properties for the closedloop system (see [22]). Let $\alpha: \Re \rightarrow \Re^{+}, \beta: \Re \rightarrow \Re^{+}$, $\gamma: \Re \rightarrow \Re^{+}$be non-negative continuously differentiable functions with $\alpha(0)=\beta(0)=\gamma(0)=0$ and such that

$$
x \alpha^{\prime}(x)>0, x \beta^{\prime}(x)>0, x \gamma^{\prime}(x) \geq 0 \text { for all } x \neq 0
$$

if $x \rightarrow \pm \infty$ then $\alpha(x) \rightarrow+\infty$ and $\beta(x) \rightarrow+\infty$

For example, $\alpha(x)$ and $\beta(x)$ may be of the form $K x^{m}$ where $K>0$ and $m>0$ is an even integer. Consider now the family of Control Lyapunov Functions:

$$
V(x)=\alpha\left(x_{1}\right)+\beta\left(x_{2}\right)+\gamma\left(e^{x_{2}}+(R-1) e^{x_{1}}-R\right)
$$

where

$$
R:=\frac{S_{0}}{s_{S}^{d e s}}
$$


For example, the selection $\alpha(x)=\beta(x):=\frac{1}{2} x^{2}$ and $\gamma(x) \equiv 0$ gives $V(x)=(1 / 2) x_{1}^{2}+(1 / 2) x_{2}^{2}$. Notice that by virtue of (27), the function $V$ is radially unbounded. The time derivative of $V$ along the trajectories of (25) is given by:

$$
\begin{aligned}
\dot{V}= & \left(\mu\left(s_{s}^{\mathrm{des}} e^{x_{2}}\right)-D\right) \alpha^{\prime}\left(x_{1}\right) \\
& +\left(D\left(R e^{-x_{2}}-1\right)-\mu\left(\mathrm{s}_{s}^{\mathrm{des}} e^{x_{2}}\right)(R-1) e^{x_{1}-x_{2}}\right) \beta^{\prime}\left(x_{2}\right) \\
& -D\left(e^{x_{2}}+(R-1) e^{x_{1}}-R\right) \gamma^{\prime}\left(e^{x_{2}}+(R-1) e^{x_{1}}-R\right)
\end{aligned}
$$

Since $\alpha^{\prime}(x)\left(e^{-x}-1\right)<0, \beta^{\prime}(x)\left(e^{-x}-1\right)<0$ for all $x \neq 0$ (a direct consequence of (26)), it is concluded from (26), (30) that the smooth feedback law:

$$
D=\mu\left(s_{s}^{\operatorname{des}} e^{x_{2}}\right) e^{x_{1}}
$$

will globally stabilize the origin for system (25). Particularly, we have:

$$
\begin{array}{r}
\dot{V}=\mu\left(s_{s}^{\text {des }} e^{x_{2}}\right) e^{x_{1}}\left[\left(e^{-x_{1}}-1\right) a^{\prime}\left(x_{1}\right)+\left(e^{-x_{2}}-1\right) \beta^{\prime}\left(x_{2}\right)\right. \\
\left.-\left(e^{x_{2}}+(R-1) e^{x_{1}}-R\right) \gamma^{\prime}\left(e^{x_{2}}+(R-1) e^{x_{1}}-R\right)\right]
\end{array}
$$

Transforming the feedback law (31) back to the original coordinates (via (24)) results in

$$
D=\frac{\mu(s) x}{Y_{x / s}\left(S_{0}-s_{s}^{\mathrm{des}}\right)}
$$

Notice that from equations (31), (32), it is guaranteed that $D \geq 0$ for all times.

The feedback law (32) is a nonlinear state feedback law and, in general, requires measurement of both biomass and substrate. However, since it has been assumed that the measured output is proportional to the product $\mu(s) x$ (see (2)), it becomes the linear output feedback law (3). It is important to point out that the control law of equation (3) coincides with the constant yield control law of [18], where the methane yield (defined as the ratio between the methane production rate and the feed flow rate) was maintained at a constant set-point value. Mailleret and Bernard [12], using a different approach, arrived at the same control law and provided a rigorous justification of global closed-loop stability.

The closed-loop system (25) under the feedback law (31) is:

$$
\begin{aligned}
\frac{\mathrm{d} x}{\mathrm{~d} t} & =\left(1-\frac{x}{Y_{x / s}\left(S_{0}-s_{s}^{\mathrm{des}}\right)}\right) \mu(s) x \\
\frac{\mathrm{d} s}{\mathrm{~d} t} & =\frac{\left(s_{s}^{\mathrm{des}}-s\right) \mu(s) x}{Y_{x / s}\left(S_{0}-s_{s}^{\mathrm{des}}\right)}
\end{aligned}
$$

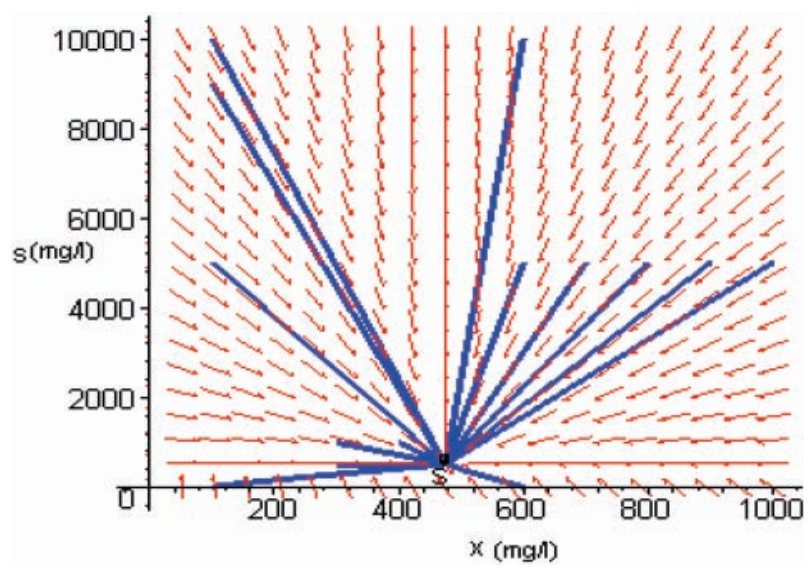

Fig. 2. Phase portrait of the closed-loop system (33).

The form of the closed-loop system shows that:

(i) the closed-loop system's equilibrium is at $x_{s}=Y_{x / s}\left(S_{0}-s_{s}^{\mathrm{des}}\right)$ and $s_{s}=s_{s}^{\mathrm{des}}$

(ii) $\frac{d\left(s-s_{s}^{\mathrm{des}}\right)}{d\left(x-Y_{x / s}\left(S_{0}-s_{s}^{\mathrm{des}}\right)\right)}=\frac{s-s_{s}^{\mathrm{des}}}{x-Y_{x / s}\left(S_{0}-s_{s}^{\mathrm{des}}\right)}$, which proves that the system's trajectories represent straight lines on the $s-x$ plane.

Figure 2 depicts a phase portrait of the closed-loop dynamics (33) for the particular parameter values and design conditions of Section 2.

\subsection{Robustness Relative to Errors in Process Parameters}

Because the output measurement $Q=Y \mu(s) x$ carries information on $\mu(s)$ and the proportional gain of the controller derived in the previous subsection is independent of $\mu(s)$, the controller is completely insensitive to errors in $\mu(s)$, in terms of achieving global stability. Of course, the computed $s_{s}^{\text {des }}$ (e.g., from equation (6)) will depend on the parameters in $\mu(s)$ (like $K_{s}$ and $K_{I}$ ) and will no longer be optimal in the presence of parameter errors. For the particular parameter values given in Section 2, which are representative of a typical anaerobic digestion process, it is found that a $50 \%$ error below the nominal values of $K_{s}$ and $K_{I}$ corresponds to 1.24 and $1.17 \%$ biogas loss, respectively and that a $50 \%$ error above the nominal values corresponds to $0.63 \%$ and $0.22 \%$ biogas loss.

The situation is different in the presence of errors in $S_{0}$. Perturbations in the organic feed are generally considered as time-varying bounded fluctuations. Therefore it is very important to investigate the situation where $S_{0}(t)$ is time-varying but bounded. Suppose that a design value $S_{0}^{\text {des }}$ is used in the control law (3), i.e., 


$$
D=\frac{1}{Y Y_{x / s}\left(S_{0}^{\mathrm{des}}-s_{s}^{\mathrm{des}}\right)} Q
$$

whereas the true feed substrate concentration in the process is $S_{0}$. Then the resulting closed-loop dynamics will follow:

$$
\begin{aligned}
& \frac{\mathrm{d} x}{\mathrm{~d} t}=\left(1-\frac{x}{Y_{x / s}\left(S_{0}^{\mathrm{des}}-s_{s}^{\mathrm{des}}\right)}\right) \mu(s) x \\
& \frac{d s}{d t}=\frac{\left[\left(S_{0}-S_{0}^{\mathrm{des}}\right)+s_{s}^{\mathrm{des}}-s\right]}{Y_{x / s}\left(S_{0}^{\mathrm{des}}-s_{s}^{\mathrm{des}}\right)} \mu(s) x
\end{aligned}
$$

In this case we define:

$$
v:=\frac{S_{0}-S_{0}^{\mathrm{des}}}{s_{s}^{\mathrm{des}}}
$$

We assume that the input $v$ takes values in the set $(-L, L)$, where $0<L<1$. Furthermore, we define:

$$
u:=\ln \left(\frac{L s_{s}^{\mathrm{des}}+S_{0}-S_{0}^{\mathrm{des}}}{L s_{s}^{\mathrm{des}}-S_{0}+S_{0}^{\mathrm{des}}}\right)=\ln \left(\frac{L+v}{L-v}\right)
$$

The input $u$ takes values in $\Re$. Notice that the closed-loop system (35) with (36), (37) under transformation (24), takes the form:

$$
\begin{aligned}
& \dot{x_{1}}=\left(1-e^{x_{1}}\right) \mu\left(s_{s}^{\operatorname{des}} e^{x_{2}}\right) \\
& \dot{x_{2}}=\mu\left(s_{s}^{\operatorname{des}} e^{x_{2}}\right) e^{x_{1}-x_{2}}\left[1-e^{x_{2}}+L \frac{e^{u}-1}{e^{u}+1}\right]
\end{aligned}
$$

In this case, we can show the following important robustness result:

Theorem 4.2: System (38) with $0<L<1$ satisfies the UISS property from the input $u$. Moreover, for every essentially bounded input $u: \Re^{+} \rightarrow \Re$, we have $\lim _{t \rightarrow+\infty} x_{1}(t)=0$.

Proof: Consider the functions:

$$
V_{1}(x)=\frac{1}{2} x_{1}^{2}, V_{2}(x)=\frac{1}{2} x_{2}^{2}
$$

and define:

$$
\begin{aligned}
& a(s)=c^{-2} s, \text { with } c>1 \\
& a_{1}(s)=\frac{1}{4} s^{2}, \quad a_{2}(s)=\frac{1}{2} s^{2}, \\
& \zeta(s)=\frac{1}{2}\left[\ln \left(\frac{e^{s}+1}{2}\right)\right]^{2}
\end{aligned}
$$

$$
\begin{aligned}
\rho_{1}(s)= & \min \left\{x_{1}\left(e^{x_{1}}-1\right) \mu\left(s_{s}^{\operatorname{des}} e^{x_{2}}\right):\right. \\
& \left.x_{1}^{2}=2 s,\left|x_{2}\right| \leq c \sqrt{2 s}\right\} \\
\rho_{2}(s)= & (1-L)^{-1} e^{-c \sqrt{2 s}} \min \left\{x_{2}\left(1-e^{-x_{2}}\right)\right. \\
& \left.\times \mu\left(s_{s}^{\operatorname{des}} e^{x_{2}}\right): x_{2}^{2}=2 s\right\}
\end{aligned}
$$

Notice that the above definitions guarantee that all requirements of Theorem 3.4 are satisfied. Particularly, implication (14) for $i=2$ follows from the inequalities:

$$
\begin{aligned}
x_{2} & >0,1+\left|\frac{\mathrm{e}^{u}-1}{\mathrm{e}^{u}+1}\right| \leq e^{x_{2}} \\
& \Rightarrow x_{2}\left(1-e^{x_{2}}+L \frac{\mathrm{e}^{u}-1}{\mathrm{e}^{u}+1}\right) \leq(1-L) x_{2}\left(1-e^{x_{2}}\right) \\
x_{2} & <0, \frac{2-e^{x_{2}}}{e^{x_{2}}} \geq e^{|u|} \geq e^{-u} \\
& \Rightarrow x_{1}\left(1-e^{x_{2}}+L \frac{\mathrm{e}^{u}-1}{\mathrm{e}^{u}+1}\right) \leq(1-L) x_{2}\left(1-e^{x_{2}}\right)
\end{aligned}
$$

in conjunction with the inequality $\left|\frac{\mathrm{e}^{u}-1}{\mathrm{e}^{u}+1}\right| \leq$ $\frac{1}{2}|u| \leq \frac{1}{2} \mathrm{e}^{|u|}-\frac{1}{2}$ (which holds for all $u \in \Re$ ) and the fact that $\frac{2-\mathrm{e}^{x_{2}}}{\mathrm{e}^{x_{2}}} \geq \mathrm{e}^{-u}$ implies $\frac{\mathrm{e}^{u-1}}{\mathrm{e}^{u+1}} \geq \mathrm{e}^{x_{2}}-1$.

We conclude that system (38) satisfies the UISS property from the input $u$ and satisfies (8) for appropriate $\sigma \in K L$ and $\gamma \in N$. Moreover, for every essentially bounded input $u: \Re^{+} \rightarrow \Re$, (8) implies that the solution is bounded over $\Re^{+}$. Consider a solution of (38) corresponding to an essentially bounded input $u: \Re^{+} \rightarrow \Re$ and let $r=\sup _{t>0}\left|x_{2}(t)\right|$. It follows that the derivative of $V_{1}(x)=(1 / 2) x_{1}^{2}$ along the solutions of (38) satisfies $\dot{V}_{1} \leq-\rho\left(V_{1}\right)$ a.e., where $\rho(s):=$ $\min \left\{\mu\left(s_{s}^{\mathrm{des}} e^{x_{2}}\right):\left|x_{2}\right| \leq r\right\} \min \left\{x_{1}\left(e^{x_{1}}-1\right): x_{2}^{2}=2 s\right\}$ is a continuous, positive definite function. Lemma 3.3 implies $\lim _{t \rightarrow+\infty} V_{1}(t)=0$ which implies $\lim _{t \rightarrow+\infty} x_{1}(t)=0$. The proof is complete.

Remark 4.3: The consequences of Theorem 4.2 are significant:

1. Estimate (8) shows that if the input $v$ takes values in a set $[-r, r]$, with $r<1$, i.e., if the error $\left|S_{0}(t)-S_{0}^{\text {des }}\right| \leq r s_{s}^{\text {des }}$, then the solution the closedloop system (35) remains in a compact set in the interior of the first quadrant in $\Re^{2}$ (Bounded Input Bounded State-BIBS property). Thus, the operation of the bioreactor cannot be led to process shut down $(\mathrm{x}=$ const., $\mathrm{s}=0, D=0)$. 
2. Estimate (8) shows that if the input $v$ takes values in a set $[-r, r]$, with $r<1$ i.e., if the error $\left|S_{0}(t)-S_{0}^{\mathrm{des}}\right| \leq r s_{s}^{\mathrm{des}}$, and if in addition we have $\lim _{t \rightarrow+\infty} S_{0}(t)=S_{0}^{\text {des }}$, then the solution of the closedloop system (35) converges to the design steady state $\left\{\begin{array}{c}x_{s}=Y_{x / s}\left(S_{0}^{\text {des }}-s_{s}^{\text {des }}\right) \\ s_{s}=s_{s}^{\text {des }}\end{array}\right\}$ (Converging Input Converging State (CICS) property).

3. Taking into account Theorem 3.4, the definitions of the functions $a_{1}, a_{2} \in K_{\infty} a, \zeta \in \mathbf{N}$ in the proof of Theorem 4.2 and transformations (24), (36), (37), estimate (8) gives for system (35):

$$
\begin{aligned}
\left|\ln \left(\frac{s(t)}{s_{s}^{\mathrm{des}}}\right)\right| \leq & \sigma\left(\left|x_{0}\right|, t\right) \\
& +\sup _{0 \leq \tau \leq t} \sqrt{2} \ln \left(\frac{L s_{s}^{\mathrm{des}}}{L s_{s}^{\mathrm{des}}+S_{0}^{\text {des }}-S_{0}(\tau)}\right)
\end{aligned}
$$

for the case $\inf _{t>0} S_{0}(t) \geq S_{0}^{\text {des }}$ and for appropriate $\sigma \in K L$. Inequality (39) shows directly the BIBS and CICS properties for the closed-loop system (35) for the case $\inf _{t \geq 0} S_{0}(t) \geq S_{0}^{\text {des }}$. Similar inequalities can be obtained for other cases.

Notice that the result of Theorem 4.2 requires essentially the following bounds for the error in $S_{0}$ :

$$
-s_{s}^{\text {des }} \leq S_{0}-S_{0}^{\text {des }} \leq s_{s}^{\text {des }}
$$

In order to investigate how sharp are the bounds (40) for the allowable error in $S_{0}$, it will be helpful to consider the special case of constant $S_{0}$. From (35) it can be easily seen that the closed-loop system's equilibrium will be at $\left\{\begin{array}{c}x_{s}=Y_{x / s}\left(S_{0}^{\text {des }}-s_{s}^{\text {des }}\right) \\ s_{s}=s_{s}^{\text {des }}+\left(S_{0}-S_{0}^{\text {des }}\right)\end{array}\right\}$, hence $x_{s}$ will equal the design value of biomass concentration, but there will be an offset of the substrate concentration from the design conditions by an amount equal to the error in $S_{0}$. A similar analysis as in Section 4.2 can show that, as long as $S_{0}-S_{0}^{\text {des }}>-s_{s}^{\text {des }}$, the closed-loop system's equilibrium is globally asymptotically stable and the trajectories of the system (35) will still be straight lines.

It is exactly the lower bound of (40) that emerges in the analysis of the case of constant $S_{0}$. This lower bound is essential because, when $S_{0}-S_{0}^{\text {des }} \leq-s_{s}^{\text {des }}$, the corresponding steady state value of the substrate, $s_{s}$, will be negative and therefore physically unrealistic. This physically unrealistic equilibrium point cannot be reached: the system trajectories will terminate on the $\mathrm{x}$-axis, which corresponds to process shut down (x= const., $s=0, D=0$ ).

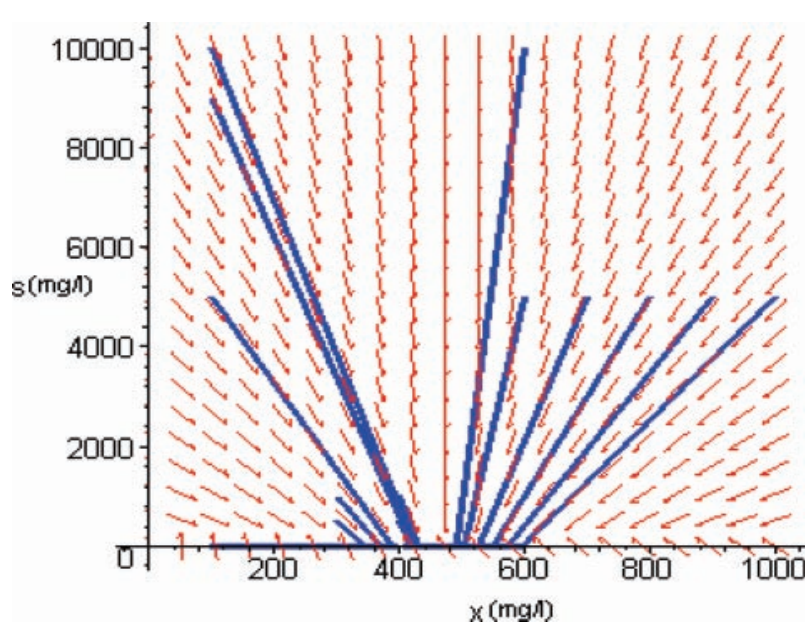

Fig. 3. Phase portrait for $S_{0}=8,000 \mathrm{mg} / \mathrm{l}$.

Figure 3 depicts the phase portrait for $S_{0}^{\mathrm{des}}=10,000 \mathrm{mg} / 1, S_{0}=8,000 \mathrm{mg} / 1$, and the rest of the parameters as in Section 2, which corresponds to $S_{0}-S_{0}^{\mathrm{des}}<-s_{s}^{\mathrm{des}}$. The controller causes the process to shut down.

The conclusion from the foregoing analysis is that the feedback control law (34) is guaranteed to be robust for any time-varying errors in $S_{0}$ that satisfy inequality (40). When the lower bound in (40) is violated (large organic feed underload), the system is brought to shut down. Shut down is completely undesirable, since it would subsequently require startup of the process, which is both time-consuming and costly.

\section{Robust Feedforward/Feedback Stabilization of the Chemostat}

If it is anticipated that the error in $S_{0}$ could be large enough to violate the robustness condition of the previous section, the control law will need to be modified to prevent shut down. One possibility is to measure the feed substrate concentration on-line and use it as a feedforward measurement in the control law. The measurement of $S_{0}$ is feasible using a Total Organic Carbon analyzer.

Consider the control law (3) or (32), where $S_{0}$ is replaced by the on-line measurement of the feed substrate concentration, i.e.,

$$
D=\frac{\mu(s) x}{Y_{x / s}\left(S_{0}-s_{s}^{\mathrm{des}}\right)}
$$

and, therefore, the control law now involves both feedback and feedforward action. Then, the resulting 
closed-loop system is still given by (33), even though now $S_{0}$ is a function of time, with $S_{0}(t)>0$ for all $t \geq 0$.

In the event that $S_{0}$ remains constant, the closedloop system's equilibrium will be at: $\left\{\begin{array}{l}x_{s}=Y_{x / s}\left(S_{0}-s_{s}^{\mathrm{des}}\right) \\ s_{s}=s_{s}^{\mathrm{des}}\end{array}\right\}$. As long as $S_{0}>s_{s}^{\mathrm{des}}$, the equilibrium will be in the first quadrant and the closed-loop system will be globally stable as a result of the analysis of Section 4.

The situation where $S_{0}(t)$ is time-varying but bounded requires further attention. Suppose that $S_{0}^{\text {des }}$ is the value of $S_{0}$ for which the reactor has been designed to operate. Then the difference

$$
v:=\frac{S_{0}-S_{0}^{\mathrm{des}}}{S_{0}^{\mathrm{des}}-S_{s}^{\mathrm{des}}}
$$

represents a bounded but, in general, non-vanishing perturbation to the system. Now assume that:

$$
|v(t)|<L, \quad \text { for all } t \geq 0
$$

where $0<L<1$ and define:

$$
u=\ln \left(\frac{L+v}{L-v}\right)
$$

Notice that the external input $u$ takes values in $\Re$. To be able to analyze the effect of this perturbation, consider the coordinate transformation:

$$
\begin{aligned}
& x_{1}=\ln \left(\frac{x}{Y_{x / s}\left(S_{0}^{\mathrm{des}}-s_{s}^{\mathrm{des}}\right)}\right) \\
& x_{2}=\ln \left(\frac{s}{s_{s}^{\mathrm{des}}}\right)
\end{aligned}
$$

Under transformation (44) and definitions (41) and (43), the closed-loop system transforms into:

$$
\begin{aligned}
& \dot{x}_{1}=\left(1-\frac{\left(e^{u}+1\right) e^{x_{1}}}{(L+1) e^{u}+1-L}\right) \mu\left(s_{s}^{\text {des }} e^{x_{2}}\right) \\
& \dot{x}_{2}=\mu\left(s_{s}^{\operatorname{des}} e^{x_{2}}\right)\left(e^{-x_{2}}-1\right) \frac{\left(e^{u}+1\right) e^{x_{1}}}{(L+1) e^{u}+1-L}
\end{aligned}
$$

In this case, we can show the following important robustness result:

Theorem 5.1: System (45) with $0<L<1$ satisfies the UISS property from the input $u$. Moreover, for every essentially bounded input $u: \Re^{+} \rightarrow \Re$, we have $\lim _{t \rightarrow+\infty} x_{2}(t)=0$.
Proof: Consider the functions:

$$
V_{1}(x)=\frac{1}{2} x_{1}^{2}, \quad V_{2}(x)=\frac{1}{2} x_{2}^{2}
$$

and define:

$$
\begin{aligned}
& a(s)=c^{-2} s, \text { with } c>1, \\
& a_{1}(s)=\frac{1}{4} s^{2}, a_{2}(s)=\frac{1}{2} s^{2}, \zeta(s)=\frac{1}{2}\left[\ln \left(1+\frac{2 L s}{1-L^{2}}\right)\right]^{2} \\
& \rho_{1}(s)=\frac{1}{2} \min \left\{x_{1}\left(e^{x_{1}}-1\right) \mu\left(s_{s}^{\text {des }} e^{x_{2}}\right): x_{1}^{2}=2 s, .\right. \\
& \left.\left|x_{2}\right| \leq c \sqrt{2 s}\right\} \\
& \rho_{2}(s)=(1+L)^{-1} e^{-c \sqrt{2 s}} \min \left\{x_{2}\left(1-e^{-x_{2}}\right)\right. \\
& \left.\times \mu\left(s_{s}^{\operatorname{des}} e^{x_{2}}\right): x_{2}^{2}=2 s\right\}
\end{aligned}
$$

Notice that the above definitions guarantee that all requirements of Theorem 3.4 are satisfied. Particularly, implication (14) for $i=1$ follows from the inequalities:

$$
\begin{aligned}
x_{1} & >0,1+\frac{2 L\left(e^{u}-1\right)}{(1-L) e^{u}+1-L} \leq e^{-x_{1}} \\
& \Rightarrow x_{1}\left(1-\frac{\left(e^{u}+1\right) e^{x_{1}}}{(L+1) e^{u}+1-L}\right) \leq \frac{1}{2} x_{1}\left(1-e^{x_{1}}\right) \\
x_{1} & <0,1-\frac{2 L\left(e^{u}-1\right)}{(1+L) e^{u}+1-L} \leq e^{-x_{1}} \\
& \Rightarrow x_{1}\left(1-\frac{\left(e^{u}+1\right) e^{x_{1}}}{(L+1) e^{u}+1-L}\right) \\
& \leq \frac{1}{2} x_{1}\left(1-e^{x_{1}}\right)
\end{aligned}
$$

in conjunction with the inequalities $\frac{2 L\left(\mathrm{e}^{u}-1\right)}{(1-L) \mathrm{e}^{u}+1+L} \leq \frac{2 L}{1-L^{2}}|u|,-\frac{2 L\left(\mathrm{e}^{u}-1\right)}{(1+L) \mathrm{e}^{u}+1-L} \leq$ $\frac{2 L}{1-L^{2}}|u|$ (which hold for all $u \in \Re$ and $0<L<1$ ).

We conclude that system (45) satisfies the UISS property from the input $u$ and satisfies (8) for appropriate $\sigma \in K L$ and $\gamma \in N$. Moreover, for every essentially bounded input $u: \Re^{+} \rightarrow \Re$,(8) implies that the solution is bounded over $\Re^{+}$. Consider a solution of (45) corresponding to an essentially bounded input $u: \Re^{+} \rightarrow \Re$ and let $r=\sup \left|x_{1}(t)\right|$. It follows that the derivative of $V_{2}(x)=1 / 2 x_{2}^{2}$ along the solutions of (45) satisfies $\dot{V}_{2} \leq-\rho\left(V_{2}\right)$ a.e., where $\rho(s)=(1+L)^{-1} e^{-r} \times$ $\min \left\{x_{2}\left(1-e^{-x_{2}}\right) \mu\left(s_{s}^{\text {des }} e^{x_{2}}\right): x_{2}^{2}=2 s\right\}$ is a continuous, positive definite function. Lemma 3.3 implies 
$\lim _{t \rightarrow+\infty} V_{2}(t)=0$, which implies $\lim _{t \rightarrow+\infty} x_{2}(t)=0$. The proof is complete.

Remark 5.2: The consequences of Theorem 5.1 are significant:

1. Estimate (8) shows that if the input $v$ takes values in a set $[-r, r]$, with $r<1$, i.e., if the error $\left|S_{0}(t)-s_{0}^{\mathrm{des}}\right| \leq r\left(S_{0}^{\mathrm{des}}-s_{s}^{\mathrm{des}}\right)$, then the solution the closed-loop system (33) remains in a compact set in the interior of the first quadrant in $\Re^{2}$ (Bounded Input Bounded State-BIBS property). Thus, the operation of the bioreactor cannot be led to process shut down $(x=$ const., $s=0, D=0)$.

2. Estimate (8) shows that if the input $v$ takes values in a set $[-r, r]$, with $r<1$ i.e., if the error $\left|S_{0}(t)-S_{0}^{\mathrm{des}}\right| \leq r\left(S_{0}^{\mathrm{des}}-s_{s}^{\mathrm{des}}\right)$, and if in addition we have $\lim _{t \rightarrow+\infty} S_{0}(t)=S_{0}^{\text {des }}$, then the solution of the closedloop system (33) converges to the design steady state $\left\{\begin{array}{l}x_{s}=Y_{x / s}\left(S_{0}^{\text {des }}-s_{s}^{\text {des }}\right) \\ s_{s}=s_{s}^{\text {des }}\end{array}\right\}$ (CICS property).

3. A comparison can be made between the feedback controller $D=\frac{\mu(s) x}{Y_{x / s}\left(S_{0}^{\text {des }}-S_{S}^{\text {des }}\right)}$ and the feedforward/feedback controller $D=\frac{\mu(s) x}{Y_{x / s}\left(S_{0}-S_{s}^{\text {des }}\right)}$ : the feedback controller guarantees that the operation of the bioreactor cannot be led to process shut down if there exists a positive constant $r<1$ such that $\left|S_{0}(t)-S_{0}^{\mathrm{des}}\right| \leq r s_{s}^{\mathrm{des}}$, whereas the feedforward/feedback controller guarantees that the operation of the bioreactor cannot be led to process shut down if there exists positive constant $r<1$ such that $\left|S_{0}(t)-S_{0}^{\mathrm{des}}\right| \leq r\left(S_{0}^{\mathrm{des}}-s_{s}^{\mathrm{des}}\right)$. As long as $S_{0}^{\mathrm{des}}>\frac{1}{2} s_{s}^{\mathrm{des}}$, the feedforward/feedback controller will have a larger guaranteed robustness margin than the feedback controller with respect to perturbations of the organic load. In practice, the operation of a bioreactor is designed so that $s_{s}^{\mathrm{des}}<<S_{0}^{\mathrm{des}}$, therefore incorporation of feedforward action will result in significant enlargement of the robustness margin. Finally, it should be noted that both control laws are completely insensitive to errors in $\mu(s)$.

Finally, comparing the result of Theorem 5.1 for time-varying $S_{0}(t)$ to the robustness results for constant $S_{0}$, we see that both results require essentially the same lower limit for $S_{0}$ :

$$
S_{0}>s_{s}^{\text {des }}
$$

The result of Theorem 5.1 also requires an upper limit $S_{0}<2 S_{0}^{\text {des }}-s_{s}^{\text {des }}$, whereas for constant $S_{0}$, there was no upper limit at all.
Comparison of Feedforward/Feedback control to pure Feedback control

Numerical simulations were performed under pure feedback action (FB) and combined feedforward and feedback action $(\mathrm{FF} / \mathrm{FB})$, when the system is initially at steady state with $S_{0}=S_{0}^{\text {des }}=10,000 \mathrm{mg} / \mathrm{l}$ but $S_{0}$ undergoes a step change to a new value. Figures 4,5 and 6 compare the responses of $x, s$ and $Q$ for some representative final values of $S_{0}$.

It is seen from Fig. 4 and 5 that, as predicted by the analysis, in a step change in $S_{0}$, the steady state of $x$ is unaffected under FB control, whereas the steady state of $s$ is unaffected under FF/FB control. The problem with FB control is when the robustness condition $s_{s}^{\text {des }}>\left(S_{0}^{\text {des }}-S_{0}\right)$ is violated, like in the case $S_{0}=$ $9,000 \mathrm{mg} / 1$, where the system is brought to shut down. For $S_{0}=9,500 \mathrm{mg} / \mathrm{l}$, the reactor is not shut down because the robustness condition is satisfied, but the final value of the substrate is very small $(s \rightarrow 6.714$

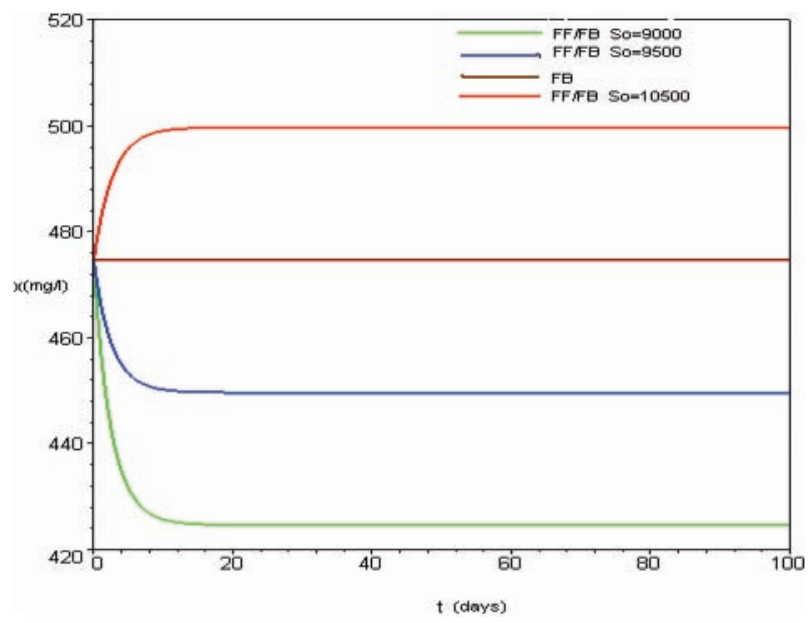

Fig. 4. Responses of $x$ for representative values of $S_{0}$.

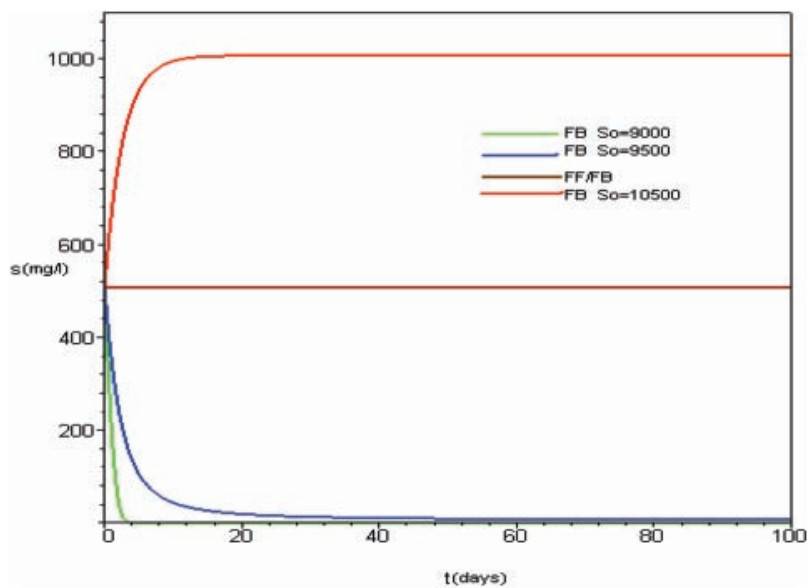

Fig. 5. Responses of $s$ for representative values of $S_{0}$. 


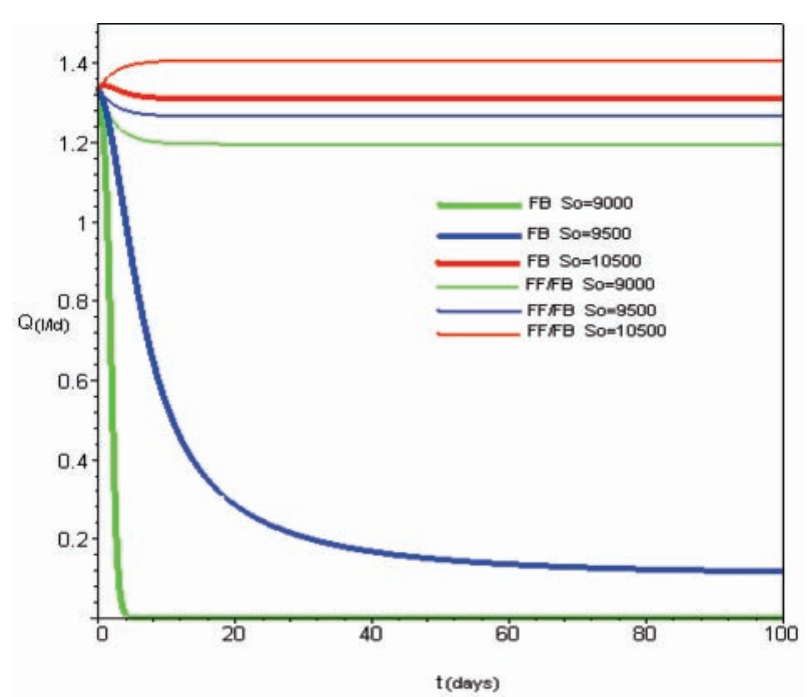

Fig. 6. Response of $Q$ for representative values of $S_{0}$.

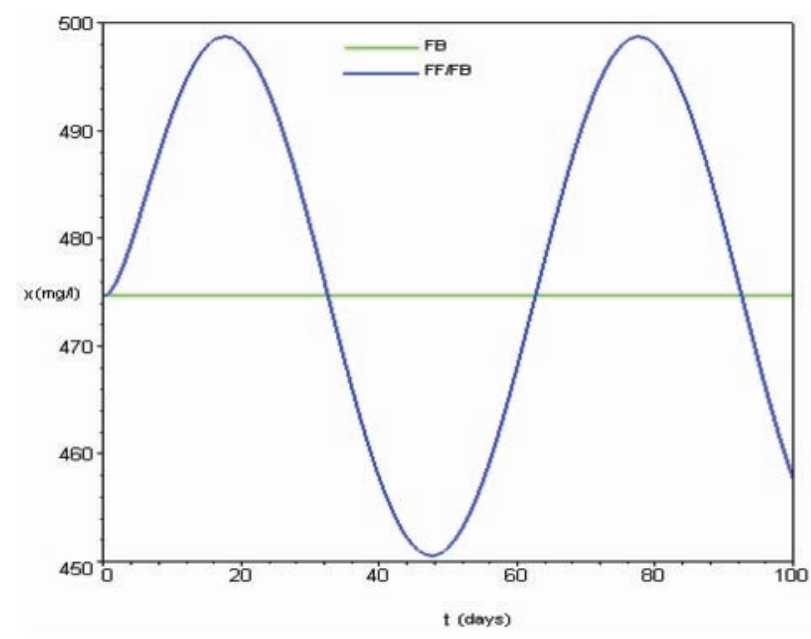

Fig. 7. Response of $x$ for a sinusoidal change in $S_{0}$.

$\mathrm{mg} / \mathrm{l})$, leading to small biogas production $\left(Q \rightarrow 0.111 l_{\text {reactor }}^{-1} d^{-1}\right)$. On the other hand, FF/FB control can tolerate much larger deviations.

In all the step changes considered, $\mathrm{FF} / \mathrm{FB}$ has better performance than $\mathrm{FB}$ in the sense that the biogas production $Q$ is larger (see Fig. 6).

Figures 7, 8 and 9 depict the responses of $x, s$ and $Q$ under a sinusoidal variation in $S_{0}$ of amplitude $500 \mathrm{mg} / 1$ and period $60 \mathrm{~d}$. The system is initially at steady state with $S_{0}=S_{0}^{\text {des }}=10,000 \mathrm{mg} / 1$ and the average value of $S_{0}$ remains the same $(10,000 \mathrm{mg} / \mathrm{l})$ for $t>0$ :

$$
S_{0}(t)=10,000+500 \sin \left(\frac{2 \pi}{60} t\right)
$$

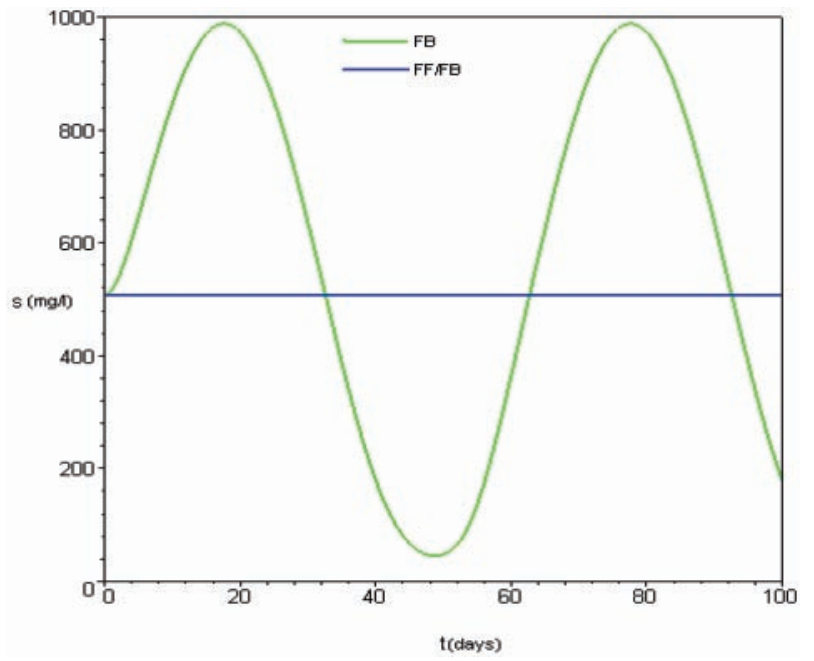

Fig. 8. Responses of $s$ for a sinusoidal change in $S_{0}$.

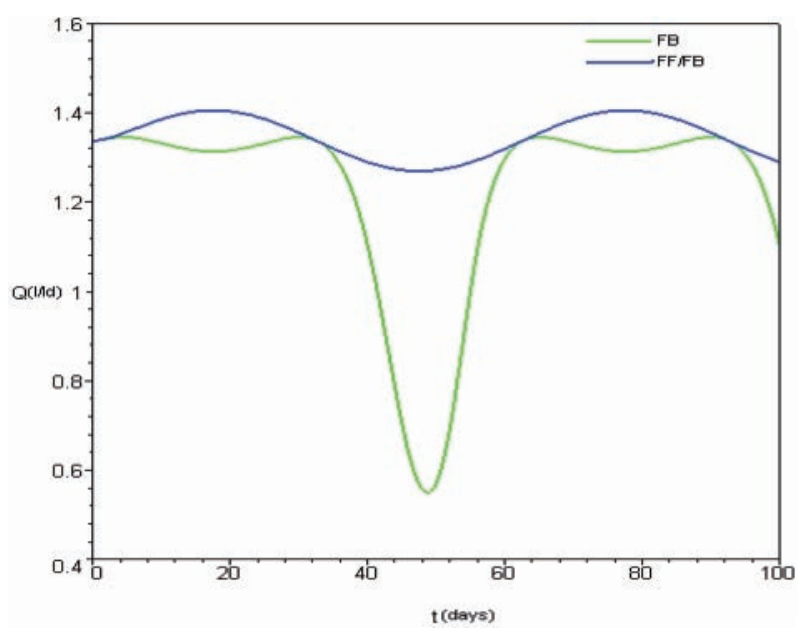

Fig. 9. Response of $Q$ for a sinusoidal change in $S_{0}$.

Here $\left|S_{0}(t)-S_{0}^{\text {des }}\right| \leq 500$, which satisfies the robustness conditions for both $\mathrm{FB}$ and $\mathrm{FF} / \mathrm{FB}$ control.

We observe that under either FB or FF/FB control, the states remain bounded and strictly positive, as predicted by the theory. Superiority of $\mathrm{FF} / \mathrm{FB}$ control is suggested in Fig. 9, in terms of higher biogas production.

\section{Conclusions}

Vector Lyapunov functions can play an instrumental role in performing robust stability analysis in terms of the UISS property. This work has developed the theoretical tools for this purpose and has applied them to the important engineering problem of robust global stabilization of the chemostat. 


\section{Acknowledgements}

Financial support from the University of Patras in the form of Caratheodory grant is gratefully acknowledged. The authors thank Dr. Katerina Stamatelatou for her assistance in the calculations of Section 2. Also, the authors thank Prof. Zhong-Ping Jiang for bringing to their attention the recent paper by Dashkovskiy et al. (2007).

\section{References}

1. Antonelli R, Astolfi A. Nonlinear controller design for robust stabilization of continuous biological reactors, In Proc IEEE Conference on Control Applications, Anchorage, AL, September 2000.

2. Bastin G, Dochain D., On-line Estimation and Adaptive Control of Bioreactors, Elsevier, Amsterdam, 1990.

3. Dashkovskiy S, Ruffer BS, Wirth FR. An ISS small-gain theorem for general networks. Math Control Signals Syst 2007; 19: 93-122.

4. De Leenheer P, Smith HL. Feedback control for chemostat models. J Math Biol 2003; 46: 48-70.

5. Freedman HI, So J.W.H., Waltman P. Chemostat competition with time delays. IMACS Ann. Comput. and Appl. Math., 5, 1/4 (12th IMACS World Congr Sci Com, Paris, July 1988), 1989; 171-173.

6. Gouze JL, Robledo G. Robust control for an uncertain chemostat model. Int J Robust Nonlinear Control 2006; 16(3): $133-155$.

7. Graef SP, Andrews JF. Stability and control of anaerobic digestion. WPCF J 1974; 46; 667-682.

8. Harmard J, Rapaport A, Mazenc F. Output tracking of continuous bioreactors through recirculation and by-pass. Automatica 2006; 42; 1025-1032.

9. Khalil HK. Nonlinear Systems, 2nd edition, Macmillan, New York, 1996.

10. Karafyllis, I, Pepe P, Jiang Z-P. Stability results for systems described by retarded functional differential equations, math.OC/0612211, in Cornell University Library 2006 (http://www.arxiv.org).

11. Karafyllis I, Jiang Z-P. A small-gain theorem for a wide class of feedback systems with control applications, SIAM J Control Optimization 2007; 46(4): 1483-1517.
12. Mailleret L, Bernard O. A simple robust controller to stabilize an anaerobic digestion process. In Proc 8th Conference on Computer Applications in Biotechnology 2001; 213-218.

13. Mailleret L, Bernard O, Steyer J.P. Robust regulation of anaerobic digestion processes. Water Science and Technology 2003; 48: 87-94.

14. Mazenc F, Malisoff M, De Leenheer P. On the stability of periodic solutions in the perturbed chemostat, math.OC/ 0612167, in Cornell University Library 2006 (http://www. arxiv.org).

15. Nersesov SG, Haddad WM. On the stability and control of nonlinear dynamical systems via vector Lyapunov functions. IEEE Trans Autom Control 2006; , 51(2), 203-215.

16. Perrier, M, Dochain D. Evaluation of control strategies for anaerobic digestion processes. Int J Adapt Control Signal Process 1993; 7: 309-321.

17. Pind PF, Angelidaki I, Ahring BK, Stamatelatou K, Lyberatos G. Monitoring and control of anaerobic reactors. In Biomethanation II (Ahring BK Ed.). Adv Biochem Eng Biotechnol 2003; 82: 135-182.

18. Pullammanappallil P, Svoronos SA, Chynoweth DP and Lyberatos G. Expert system for control of anaerobic digesters. Biotechnol Bioeng 1998; 58: 13-22.

19. Smith H, Waltman P. The theory of the chemostat. Dynamics of Microbial Competition, Cambridge Studies in Mathematical Biology, 13. Cambridge University Press, Cambridge, 1995.

20. Switzenbaum MS, Giraldo-Gomez E, Hickey RF. Monitoring of the anaerobic methane fermentation process. Enzyme Microb Technol 1990; 12: 722-730.

21. Sontag ED. Smooth stabilization implies coprime factorization. IEEE Trans Autom Control 1989; 34: 435-443.

22. Sontag ED. Mathematical Control Theory. Deterministic Finite-Dimensional Systems, $2^{\text {nd }}$ edition, Springer-Verlag, New York, 1998.

23. Sontag ED, Ingalls B. A small-gain theorem with applications to input/output systems, incremental stability, detectability, and interconnections. J Franklin Inst 2002; 339: 211-229.

24. Vorotnikov VI. Partial Stability and Control. Birkhauser, Boston, 1998.

25. Wolkowicz G, Xia H. Global asymptotic behavior of a chemostat model with discrete delays. SIAM J Appl Math 1997; 57: 1019-1043.

26. Wang L, Wolkowicz G. A delayed chemostat model with general nonmonotone response functions and differential removal rates, J Math Anal Appl 2006; 321: 452-468. 